\title{
Analysis of Pre-Erythrocytic Immunity During Plasmodium Vivax Infection Reveals a Diversity of Responses That is Partially Due to Blood Stage Cross-Reactivity
}

Julio Ventocilla

Naval Medical Research Unit No 6 https://orcid.org/0000-0001-5648-1454

\section{Lorena Tapia}

Naval Medical Research Unit No 6

Lisa Sperling

Naval Medical Research Unit No 6

\section{Reynaldo Ponce}

Naval Medical Research Unit No 6

\section{Adriano Franco}

Naval Medical Research Unit No 6

\section{Mindy Leelawong}

NYC DOHMH: New York City Department of Health and Mental Hygiene

Joao C. Aguiar

NMRC: Naval Medical Research Center

G. Christian Baldeviano

Bluebird bio, Inc

Brandon K. Wilder ( $\nabla$ brandon.k.wilder2.civ@mail.mil )

Naval Medical Research Unit No 6 https://orcid.org/0000-0002-5577-7787

\section{Research}

Keywords: Plasmodium vivax, pre-erythrocytic stage, Aotus nancymaae, cross reactivity, natural acquired immunity, Peruvian amazon Basin.

Posted Date: May 24th, 2021

DOl: https://doi.org/10.21203/rs.3.rs-518437/v1

License: (9) (1) This work is licensed under a Creative Commons Attribution 4.0 International License. Read Full License 


\section{Abstract}

\section{Background}

Plasmodium vivax $(P v)$ represents the most geographically widespread human malaria parasite.

Targeting the pre-erythrocytic (PE) stage of the parasite life cycle is especially appealing for $P v$ vaccines as it would prevent disease and transmission. Here, we explore naturally acquired immunity to a panel of $P V$ PE antigens as a first step to enable vaccine development and to better understand naturally-acquired PE immunity.

\section{Methods}

Humoral and cellular immunity were evaluated by ELISA and ELISpot, using samples from $P v$ infected individuals from a low endemic malaria region in the Peruvian Amazon Basin. In addition, we utilized experimental infection of Aotus non-human primates with $P v$ or $P$. falciparum (Pf) in order to evaluate the contribution of blood stage infection to the humoral response observed in human samples.

\section{Results}

In our clinical samples, twelve PE antigens showed positive antibody reactivity with variable prevalence of $58-99 \%$. The magnitude of the IgG antibody response against PE antigens was lower compared with blood stage antigens MSP1 and DBP-II although titers persisted better for PE antigens, six months later after infection (average decrease of $6 \%$ for PE antigens and $43 \%$ for MSP1) in general. A significant correlation between IgG antibodies and number of previous malaria episodes was observed only for blood stage antigens. High IgG responders across PE and blood stage antigens showed a significantly lower parasitemia compared to low responders (median 1873 vs $4663 \mathrm{par} / \mu \mathrm{l}$ ). We observed a positive T cell response in $35 \%$ vs $9-35 \%$ of total volunteers against blood stage antigen MSP1 and PE antigens, respectively, and saw no correlation with IgG responses. Aotus monkeys infected with $P v$ blood stage showed positive reactivity against the seven PE antigens tested. In contrast, only 2 of 10 monkeys infected with $P f$ showed low positive IgG cross-reactivity against $P v \mathrm{MSP} 1$ and none of which crossreacted to $P V$ CSP.

\section{Conclusions}

Our results demonstrate clear humoral and T cell responses against $P V P E$ antigens in individuals naturally infected with $P$. vivax. In addition, these results are largely replicated in a novel Aotus nancymaae $P v$ blood stage challenge model which suggest a contribution from blood stages to PE crossreactivity. Together, these data clearly identify novel attractive PE antigens suitable for use in the development of new malaria vaccine candidates.

\section{Background}


Plasmodium spp. is the causative agent of malaria and one of the world's deadliest infectious diseases. In 2018 about 228 million cases of malaria were reported by WHO with approximately 405,000 deaths, particularly in young children and pregnant women in sub-Saharan Africa[1]. While Plasmodium falciparum $(P f)$ is the most prevalent malaria parasite on the African continent and responsible for most observed infections and deaths, $P$. vivax $(P v)$ represents the most geographically widely distributed human malaria parasite worldwide. Outside of Africa, $P$. vivax is the dominant cause of malaria with over 3 billion people living within its transmission limits[1]. Globally, several million clinical cases of $P$. vivax malaria are detected each year with a relevant portion in South East Asia and Latin America, where $P v$ is responsible for $50 \%$ and $70-85 \%$ of all malaria cases, respectively[1]. Traditionally, P. vivax has been considered to cause a "benign" form of malaria but is now recognized as a significant cause of morbidity and mortality due to increasing evidence of severe cases with a possible fatal outcome[2].

Malaria elimination efforts have resulted in a substantial decline in the global malaria burden in the past two decades. It is estimated that these efforts have resulted in a reduction of global malaria infections by $29 \%$ and mortality caused by malaria by $60 \%$ between 2000 and 2018[1]. However, from 2014 to 2018 this downward trend has slowed considerably and even reversed in some regions $[1,3]$. P. vivax represents a special challenge to control efforts due to its unique biological features, including low-density bloodstage infection, asymptomatic infections and the formation of hypnozoites, which are dormant forms residing in hepatocytes that can cause relapses months or years after the initial infection. Hypnozoites are believed to be responsible for approximately $80 \%$ of infections and there is currently no diagnostic tool available for their detection[4]. Further progress towards malaria elimination will require additional tools including the development of an effective vaccine or intervention which can prevent or eliminate these dormant stages. Targeting the pre-erythrocytic (PE) stage-the asymptomatic stages from the skin to liver which precede the symptomatic blood (erythrocytic) stage-of the parasite is especially appealing for $P$. vivax vaccine development as it would prevent disease arising from both primary and relapse infection.

For many years, the efforts to develop a PE malaria vaccine have mainly been focused on $P$. falciparum while only two $P$. vivax vaccine candidates have reached clinical trials for $P$. vivax (targeting blood stage proteins DBPII and MSP1)[5]. In contrast, several vaccine candidates against $P$. falciparum are under development. The most advanced of these is the subunit vaccine RTS,S-AS01 which targets the Circumsporozoite protein (CSP) via neutralizing antibodies and has shown consistent but short-lived efficacy in children in phase 3 clinical trials[6, 7]. In addition to CSP, other proteins have been under investigation as vaccine targets, most notably the $\mathrm{PE}$ antigens thrombospondin-related adhesive protein (TRAP) and the highly promising blood stage protein reticulocyte-binding protein Homolog 5 (RH5)[8]. The latter was chosen based on association with protection in naturally infected persons and is progressing into clinical trials after encouraging preclinical results in non-human primates $[9,10]$.

Only a few such studies of naturally acquired immunity to $P$. vivax have been conducted, largely with a focus on antibodies to erythrocytic antigens, which has limited our selection of PE antibody targets mostly to orthologs of $P f$ candidates. In these studies, reticulocyte-binding proteins critical for merozoite 
invasion have been associated with protective immunity (e.g. PVDBPII and PVRBP2b)[11-14]. Large-scale screenings have identified antibodies to other blood-stage antigens associated with protection particularly when seen in combinations rather than with any single antigen alone[15, 16]. However, even fewer studies have explored the immunogenicity of $P V P E$ antigens[17] despite the fact that they can be targeted potentially with both antibodies and $T$ cells $[18,19]$. Furthermore, targeting this stage to prevent or eliminate hypnozoites would have an outsized effect on elimination given that relapse is the main driver of disease and transmission[4].

Taken together, evidence from $P f$ and $P v$ indicate that antibodies and T cells targeting multiple PE $P v$ proteins could be an integral part of the first effective $P v$ vaccine. The almost exclusive focus on the blood stage has resulted in a general lack of $P v P E$ candidate antigens and an even more opaque understanding of their role in natural immunity or even simply immunogenicity. This paucity of data around PE immunity in $P v$ limits our ability to take the first steps towards novel vaccine candidates. Here, we explore naturally acquired immunity to a panel of $P V P E$ antigens as a key step to enable vaccine development and to better understand naturally-acquired PE Pv immunity.

\section{Methods}

\section{Human plasma and lymphocyte samples}

Human samples were obtained from three protocols approved by the Institutional Review Board of the U.S. Naval Medical Research Unit No.6 (NAMRU-6) in compliance with all applicable federal regulations governing the protection of human subjects (NMRCD.2007.0004, NMRCD.2010.0002 and NAMRU6.2012.0006). These studies were conducted at peri-urban health centers of Hospital Regional de Loreto (HRL, $n=41$ ) and Hospital de Apoyo de lquitos (HAl, $n=35$ ) between 2012 and 2017. Subjects were enrolled by passive malaria surveillance of patients presenting malaria symptoms at both health centers located in Iquitos, the largest city in the Peruvian Amazon Basin. This area is described as low $P$. vivax endemic with annual average cases of 42,164 \pm 7,779 during 2012-2017, although heterogeneous and sustained local $P$. vivax transmission has been reported[20], resulting in underestimated malaria prevalence due to low-parasitemia and/or asymptomatic cases. All subjects enrolled were diagnosed by microscopy and confirmed by PCR[21] for detection of $P v$ mono-infection. Additionally, we used twentyfour samples of $P$. vivax infected patients for long-term analysis with 6 months follow-up enrolled at NMRCD.2010.0002 and NAMRU6.2012.0006 protocols. A set of twenty human P. vivax negative control blood samples were obtained from individuals living in the department of Piura, located in the North Coast of Peru (NMRCD.2010.0002), which has reported very low incidence of $P$. vivax malaria (0.01 per 1000 inhabitants) during 2014.

\section{Aotus nancymaae infection and plasma samples}

The experiments reported herein were conducted in compliance with the Animal Welfare Act and in accordance with principles set forth in the "Guide for the Care and Use of Laboratory Animals," Institute of 
Laboratory Animals Resources, National Research Council, National Academy Press, 2011. All animals were bred in captivity and included in NAMRU-6 IACUC approved protocols (NAMRU-6 13 - 04, NAMRU-6 11-12 and NAMRU-6 14-06). In addition, these animal studies were approved by the Peruvian government agency of "Servicio Nacional Forestal y de Fauna Silvestre" (SERFOR) with permit codes: RDG: 096-2014-SERFOR-DGGSPFFS, RDG: 184-2012-AG-DGFFFS and RDG: 050-2017-SRFOR-DGGSPFFS.

We obtained plasma samples from Aotus nancymaae monkeys $(n=19)$ between days 3 to 39 post blood stage infection with $P$. vivax or $P$. falciparum to evaluate immunogenicity against $P$. vivax $\mathrm{PE}$ antigens.

We used plasma samples from $A$. nancymaae monkeys $(\mathrm{n}=10)$ infected with $P$. falciparum belonged to control groups used in $P$. falciparum pre-clinical trials of RH5 vaccine efficacy performed previously at NAMRU-6 (NAMRU-6 11-12 and NAMRU-6 $14-06$ ) [9, 10]. For these, one vial of cryopreserved infected red blood cells (iRBCs) of $P$. falciparum FVO strain was inoculated to a donor monkey and once parasitemia was between $2,000-10,000 \mathrm{par} / \mu \mathrm{l}$, this blood was used to infect study animals with a dose of $1 \times 10^{4}$ par/animal. These monkeys were followed up by microscopy detection of parasitemia for 39 days post-infection. This group did not receive any RH5 vaccine and thus developed natural parasite kinetics requiring treatment with Mefloquine (MQ) when parasite density reached $>200,000 \mathrm{par} / \mu \mathrm{l}$ in peripheral blood or when hematocrit fell below $25 \%$.

Plasma samples from monkeys infected with $P$. vivax were collected from our current work in the development of a $P$. vivax blood stage infection Aotus nancymaae model. Briefly, cryopreserved iRBCs from $P$. vivax Vietnam IV line were used to infect a donor monkey and once parasitemia at peripheral blood was between $2,000-10,000 \mathrm{par} / \mu \mathrm{l}$ this blood was used to infect spleen-intact monkeys with dose of $2.5 \times 10^{6} \mathrm{par} / \mathrm{animal}(\mathrm{n}=3)$ or $1.0 \times 10^{6} \mathrm{par} / \mathrm{animal}(\mathrm{n}=6)$. Parasitemia and plasma samples were assessed for 39 days post- infection by microscopy. Treatment with Chloroquine (CQ) was initiated when parasite density reached $>250,000 \mathrm{par} / \mu \mathrm{l}$ in peripheral blood or when hematocrit fell below $25 \%$.

\section{Selection of P. vivax PE antigens for immunological assessments}

The $13 P V$ PE antigens are orthologs to a panel of $P$. falciparum antigens previously identified as PE vaccine targets[22]. These $P f$ antigens were selected from a larger set of $131 P f$ recombinant proteins based on their reactivity against sera samples from sterilely protected subjects who underwent immunization with radiation attenuated sporozoites (RAS) followed by challenge with the bites of viable $P f$-infected Anopheles mosquitoes[23, 24]. This approach has been partially validated for three $P$. yoelii orthologs from this list of candidates: a putative cysteine protease inhibitor (Falstatin), the gamete egress and sporozoite traversal (GEST) and the early transcribed membrane protein (ETRAMP) which induced up to $60 \%$ protective efficacy when used in combination using the DNA and vaccinia virus prime-boost immunization approach[25]. In addition, our subset of $P v$ proteins have been verified as expressed in a published in P. vivax proteome[26].

These proteins were successfully expressed by the cell-free wheat germ expression system[27] using the bi-layer method (small-scale) with a yield between 300-1200 ug of protein per reaction. 


\section{ELISA - human samples}

Briefly, thirteen $P V$ PE antigens and two blood stage antigens were used to determine antibody prevalence and relative immunogenicity. ELISA plates were coated with recombinant proteins at concentration of 2$4 \mathrm{ug} / \mathrm{ml}$ to high-binding 96-well microplates (Nunc Maxisorp) overnight at room temperature and then washed with T-PBS1X (PBS1X/0.05\%Tween20) five times. Microplates were blocked for 1 hour with TBS1X-SM (TBS1X/Skim milk 5\% buffer). After five washes with T-PBS1X, human plasma samples were added in duplicate at a 1:200 dilution in TBS1X-SM and incubated for 2 hours at room temperature. After five washes with T-PBS1X, antibodies were detected using peroxidase-conjugated anti-human IgG monoclonal antibodies (Jackson Immunoresearch Cat\#: 309-035-033, 1:6,000 dilution) and incubated for one hour at room temperature. After five washes with T-PBS1X, we used o-phenylenediamine (Sigma Aldrich Cat\#: P3804) with hydrogen peroxide as a substrate and the reaction was stopped after 1 hour with $50 \mathrm{ul}$ of $3 \mathrm{~N} \mathrm{HCl}$. Plates were read at $492 \mathrm{~nm}$ to determine optical density OD.

A two-fold plasma serial dilution of a positive pool of 30 individuals with more than 10 P. vivax confirmed events were used as standard curve and included on each plate run. In addition, for positivity cut-off calculation we used 20 plasma samples from individuals from malaria area with low incidence to calculate a cut-off value for each protein using the average OD value plus 3 standard deviations.

\section{ELISA - Aotus nancymaae samples}

The immunogenicity of $P V P E$ antigens following blood stage infection of $A$. nancymaae monkeys was assessed for seven $P V$ PE antigens one Blood stage antigen similar to our human ELISA. Briefly, 96-well microplates (Nunc Maxisorp) were coated with $4 \mu \mathrm{g} / \mathrm{ml}$ of each $P v$ protein and incubated overnight at room temperature. After washing, plates were blocked for 1 hour with TBS1X-SM and monkey plasma samples were then added at 1:100 in TBS1X-SM and incubated for 2 hours at room temperature.

Antibodies were detected using peroxidase-conjugated anti-monkey IgG (Sigma Aldrich Cat\#:A2054) with an incubation of 1 hour. 0-phenylenediamine (Sigma Aldrich Cat\#: P3804) with hydrogen peroxide was used as substrate and the reaction was stopped after 1 hour with $3 \mathrm{~N} \mathrm{HCl}$, then plates were read at 492 $\mathrm{nm}$.

\section{ELISpot}

A library of overlapping 15-mer synthetic peptides was obtained from Mimotopes Pty Ltd. The peptides overlapped by 9 aminoacids spanning the entire protein. PvMSP-1 was used as positive control of blood stage antigens, and seven pre-erythrocytic antigens (CSP, CelTOS, Falstatin, ETRAMP, PVX_119755, GEST and HSP PVX_089585). These were resuspended according to the manufacturer instructions and each peptide pool used at $10 \mu \mathrm{g} / \mathrm{ml}$. These peptides were used to determine the frequency of T-cell response producing IFN-y to Pv PE proteins using Human IFN-y ELISpot PRO (Cat.\# 3420-2APW-10. Mabtech AB, Sweden) according to the manufacturer's instructions. 
Peripheral blood mononuclear cells (PBMCs) from $P$. vivax patients were isolated from whole blood by density centrifugation using a Percoll gradient, counted and cryopreserved for storage in liquid nitrogen. Briefly, PBMCs were thawed in media with $10 \%$ FBS (SIGMA, F4135), incubated overnight at $37^{\circ} \mathrm{C}$ and then plated at $0.2 \times 10^{\wedge} 6$ cells $/$ well in duplicates. Peptides were added at $10 \mathrm{ug} / \mathrm{ml}$ to stimulate $T$ cell response for 18 hours with PMA/ION used as positive stimulation control. Spots were counted using the CTL IMUNOSPOT Analyzer. Subjects were defined as positive when the number of spots was higher than the negative control by $20 \%$.

\section{Statistical analysis}

Analysis was performed using STATA v16.0 statistical software (Stata Corp., College Station, TX, USA) and GraphPad Prism v9 (GraphPad Software, LLC). Differences among the frequency of epidemiological and immunological variables were analyzed using Chi-square test for categorical variables and MannWhitney or Kruskal-Wallis test for numerical continuous variables to compare the median values. Spearman rank correlation coefficient test was used to evaluate the correlation between epidemiological and immunological variables. To measure differences of antibodies level at different time points between groups (related samples) per each antigen we used Wilcoxon signed-rank test, the significance level for all statistical analysis performed was set at $p<0.05$ or $p<0.001$.

\section{Results}

\section{Epidemiological variables}

Epidemiological variables by site of enrollment showed significant differences only for weight and number of previous $P$. vivax episodes (Table 1). Both variables were higher for patients enrolled at Hospital Regional de Loreto (HRL).

\section{Human plasma samples fromP. vivaxinfected population and humoral response}

In order to determine if antibodies recognizing $P v P E$ antigens are induced during naturally occurring $P V$ infections, we tested plasma samples from up to $76 P v$ positive patients enrolled at health centers of HRL and HAI during 2012-2017 by ELISA.

This group of 26-76 Pv patients showed positive antibody reactivity with high prevalence for blood stage antigens MSP1 (92\%), DBP-II (79\%) and the PE stage antigen CSP (99\%) (Fig. 1A). Twelve other PV PE antigens showed positive antibody reactivity with variable prevalence in the range of 58-99\%. Prevalence for each antigen was: TRAP PVX_082735 (99\%), HSP PVX_089585 (93\%), GAP40 PVX_08046 (93\%), Hypothetical protein PVX_119755 (93\%), CELTOS PVX_123510 (91\%), GEST PVX_121950 (89\%), SPECT1 PVX_083025 (89\%), FALSTATIN PVX_09903 (88\%), Hypothetical protein PVX_094725 (75\%), Hypothetical protein PVX_111090 (75\%), ETRAMP (UIS3 ortholog) PVX_121950 (72\%), and Hypothetical protein PVX_093660 (58\%) (Fig. 1). 
The magnitude of the antibody response as measured by OD values was higher for the blood stage MSP1 antigen with an OD average of 1.8 compared to the canonical PE antigen CSP with an OD average of 0.8. The other $12 P V P E$ antigens showed variable intensity with a mean OD range of 0.3-0.7. (Fig. 1). Overall, the antibody magnitude did not correlate with parasitemia levels at the time of sampling except for a modest negative correlation in 5 PE antigens (Spearman RHO: -0.23 to $-0.39, p<0.05$ ) (Table S1).

To determine if antibody magnitude correlated with previous malaria exposure, we used a subgroup of 59 patients who self-reported previous malaria episodes, which we stratified by no previous $(n=26)$, one previous $(n=16)$ and two or more previous $(n=17)$ P. vivax episodes. There was a significant increase of IgG antibodies against only the blood stage antigens MSP1 and DBP in groups with more than one previous episodes as compared to the group with no previous $P$. vivax infection (Fig. 2). IgG antibodies against all $P V P E$ antigens showed similar IgG levels independent of the number of self-reported previous malaria episodes (Fig. 2). Together, these data reveal a broad and variable seropositivity to multiple PV PE antigens during acute $P$. vivax infection that, unlike blood stage antigens, appear not to be boosted by multiple previous blood stage infections.

Antibody magnitude also showed substantial variability between volunteers, especially amongst preerythrocytic antibodies (Fig. 3). Some volunteers responded broadly to nearly every antigen while others appeared to have weak antibody responses in terms of both breadth and magnitude. Indeed, a common predictor of antibody response to one PE antigen was a response to another (Fig.S2) suggesting that some volunteers naturally respond to infection with a greater antibody response. Those "high responders", defined simply as those in the top $50 \%$ of total IgG magnitude across all antigens were evaluated against epidemiological variables. We did not observe any significant differences between high and low responders by place of sample collection, sex, age, weight, temperature and number of previous $P$. vivax episodes. However, high responders showed a significantly lower parasitemia compared to low responders (median par/ $\mu 1873$ vs $4663, p<0.05$ ) at time of enrollment (Table 2). Interestingly, high and low antibody responders did not differ in parasitemia levels after stratification by number of previous episodes (Fig.S3 A and B).

Long-term analysis of a sub-group of $24 P$. vivax patients at 6 months after enrollment showed a significant decrease of OD values only for MSP1 (OD average decrease of $-43 \%$, Day 0: 1.71 vs Day 180: $0.97, p<0.05)$ and CSP (OD average decrease of $-28 \%$, Day $0: 0.83$ vs Day $180: 0.60, p<0.001)$. Nine PE antigens showed more stable IgG levels with an average decrease of between antigens of $6 \%$ (range: -2 to -21 and $S D \pm 6 \%$ ) (Fig. 4), indicating that some PE antibodies are maintained for a significant period after infection.

\section{Human T cell responses}

We next evaluated the T cell PE immune response in a small set of individuals $(n=17)$ for which we had PMBCs. Ex vivo IFN-gamma response was detected in at least one subject for all of the antigens tested. Positivity was the highest for the blood stage protein MSP1 and the pre-erythrocytic stage protein CSP, both at $35.3 \%$ or $6 / 17$ volunteers positive. From the remaining antigens tested, the highest percentage of 
positive responses was for ETRAMP at 33.3\% (5/15), followed by Falstatin $(25 \%, 4 / 16)$, CelTOS $(23.5 \%$, 4/17), the Hypothetical protein PVX_119755 (23.1\%, 3/13) and HSP (18.2\%, 2/11).

Across individuals, we observed broad and variable T cell responses to PE antigens (Fig. 5) with 2 subjects positive to CSP only, and 6 subjects positive to 2-4 PE antigens (in addition to or besides CSP). Variability between subjects in the number of SFU (spot forming units) per million cells was also seen, with two subjects highly reactive to multiple antigens. Additionally, two subjects were positive to only MSP1 and no T cell response was found for seven subjects.

We were able to assess the antibody response in seven of the subjects for which we had both plasma and PBMCs. In this limited set, there was no apparent correlation between positive IgG response and ex vivo IFN-gamma response (Table 3). Together, these data indicate that $\mathrm{T}$ cell responses to both blood stage and PE antigens are also present but variable during acute $P v$ infection.

\section{Aotus nancymaaeas a model for studyingP. vivaxblood stage infection and the humoral immune response}

The study of $P$. vivax immunology is severely limited by the lack of appropriate animal or human challenge models in which to perform controlled infections. This complicates analysis of exact infection and immune kinetics especially in the context of discerning the relative contribution of primary vs. relapse infection. Existing animal models in Aotus and Saimiri new world monkeys typically require splenectomy to observe consistent infection which precludes study of the subsequent immune response. To address this, we tested a strain of $P$. vivax previously reported to elicit robust infection in Aotus new world monkeys for its ability to produce reliable infection in spleen-intact animals. $A$. nancymaae monkeys were inoculated with $2.5 \times 10^{6}$ (Experiment 1 ) or $1 \times 10^{6}$ (Experiment 2) infected red blood cells (iRBC) /animal of $P$. vivax Vietnam-IV strain (Fig. 6A). These animals showed low variation between experiments 1 and 2 for day of first parasitemia or "patency" ( $5 \pm 1$ vs $6 \pm 1$ ), and day of maximum parasitemia (13 \pm 2 vs $14 \pm 2$ ). We observed a numerically higher average of maximum parasitemia in experiment $2(41,747 \pm 68,672$ par/ul) compared with experiment $1(16,400 \pm 3735 \mathrm{par} / \mathrm{ul})$ but this was not statistically significant $(\mathrm{p}=$ 0.714 ) and was driven largely by a single animal which had a peak of 194,000 parasites/ $\mu \mathrm{L}$ compared to a range of 2,100 - 29,200 parasites/ $\mu \mathrm{L}$ for the remaining animals (Fig. 6A and Table 4). Monkeys from both experiments had an average of 14 days of detectable parasitemia and all self-cured parasitemia on average at day $24 \pm 3$ post-infection. In summary, infection of spleen-intact $A$. nancymaae new world monkeys with $P$. vivax Vietnam IV demonstrates $100 \%$ infection with variable parasitemia in the ranges as seen during human infection.

Plasma samples from these animals were used to measure IgG to a subset of our $P$. vivax antigens during the course of $P$. vivax blood stage infection.

All $P$. vivax Vietnam-IV blood stage infected monkeys showed positive reactivity against the seven $P v P E$ antigens tested. We observed antibody peak between days 21-28 post-infection which was approximately 1-2 weeks post-peak parasitemia (Fig. 6B). 
To evaluate the relationship between parasitemia and IgG antibody response against $P V P E$ antigens we calculated Area Under the Curve (AUC) for both variables. Comparison of AUC of parasitemia or peak parasitemia with antibodies during the follow-up period showed variable response with no clear correlation between either parasitemia or IgG response (Fig. 6C-D). Individual IgG responses and parasitemia for each animal are detailed in Fig S4. Together, these data indicate that the $P v$ blood stage infection induces antibodies to both erythrocytic and PE antigens which vary between individual animals in a manner that does not correlate with parasitemia but rather with the incidence of other anti-parasite antibodies.

The IgG reactivity to PE antigens following blood stage infection showed a correlation with MSP1 antigen (Pearson: $0.86-0.94, p<0.05$ ) as well as with all other antigens tested (Fig. 6E). Together, this clearly demonstrates the expression or cross-reactivity of $\mathrm{PE}$ antigens during blood stage infection where, like our human cohort, an antibody response to one antigen correlates with the response to others (Fig. 6B and $C-D$ ).

\section{Cross-reactivity of IgG antibodies betweenP. vivaxandP. falciparuminfections.}

Given that $A$. nancymaae monkeys can also be infected with $P$. falciparum blood stages, this offers the unique opportunity to investigate the cross-reactivity of $P f$ and $P v$ antigens in a controlled animal model. To this end, we used plasma samples from $A$. nancymaae monkeys used as infectivity controls in previously published $P f$ infection studies $[9,10]$.

These A. nancymaae monkeys infected with $P$. falciparum FVO showed consistent infection kinetics between experiments for prepatency day ( $6 \pm 1$ vs $6 \pm 1$ ) and day of maximum parasitemia (12 \pm 1 vs $11 \pm$ 1) (Fig. 7A and Table 5). Monkeys from both experiments were treated after counting a parasitemia over $200,000 \mathrm{par} / \mathrm{ul}$ (day of maximum parasitemia).

All animals had positive reactivity against $P$. falciparum MSP1 antigen with peak antibody day of 21, while only 2 of 10 monkeys had IgG which cross-reacted with PV MSP1 and none of which cross-reacted to $P v$ CSP. In summary, in a controlled $P f$ mono-infection of spleen intact $A$. nancymaae new world monkeys, we saw minimal cross-reactivity between blood stage antigen MSP1 and none to CSP between $P f$ and $P V$, indicating that $P E$ antigens may be better at distinguishing species-specific infections in serological surveys.

\section{Discussion}

Few $P$. vivax PE candidates have been fully evaluated and those that have are still at the pre-clinical or early clinical stages. New $P V P E$ candidates and in particular new antigens will be necessary to obtain a better understanding of $P V P E$ immunity and to provide insight into their role in the prevention of both liver and blood stage infection. In the absence of well-established animal models of $P$. vivax, studying the acquisition of $\mathrm{PE}$ antibodies during natural infection is a logical starting point with the long-term goal of 
studying the role of PE antibodies in functional immunity to justify integration into novel vaccine candidates. However, to date, few studies of this kind have focused on PE antibodies specifically.

A P. vivax cohort study in a low-transmission $P$. vivax area in western Thailand during 2013 showed prevalence of $86 \%, 21 \%$ and $0 \%$ for CSP, CELTOS and TRAP antigens, respectively[28]. However, a crosssectional study of naturally acquired immunity performed in Brazil during 2016 against whole and synthetic peptides of PE TRAP showed prevalence of $46 \%$ and $25-32 \%$, respectively[29]. Our results showed naturally acquired immunity against all $12 \mathrm{PE}$ antigens tested in $P$. vivax patients with generally higher proportions of individuals responding to all PE antigens (range of 58-99\%). This variation could be related to the inherent characteristics of different circulating $P$. vivax parasites, differences in transmission dynamics or simply differences in protein production platforms as these previous studies used HEK-293T cells compared to our wheat germ Cell-free system. Compared to the magnitude of blood stage responses, the IgG response to PE antigens was lower. This is likely due to the fact we did not see a boosting effect of anti-PE IgG with repeated infection.

Interestingly, we found IgG responses to one antigen often correlated simply with a response to another antigen within individuals-leading to high and low antibody responders. Comparative analysis of high and low antibody responders against $P v \mathrm{PE}$ and blood stage antigens vs. parasitemia showed that high responders had lower parasitemia levels $(\mathrm{p}<0.05)$. In addition, we observed that the low parasitemia in high responders was similar even through multiple of previous $P$. vivax episodes. Whether this is a result of a diverse antibody response better controlling multiple infections, from a low parasitemia exerting less suppressive effects on the antibody response[30] or is due to inherent individual differences between individuals which dictate broad reactivity[31] are questions which warrant further investigation in welldesigned longitudinal cohort studies.

Stability analysis of IgG antibodies six months after $P$. vivax infection showed a significant decrease of antibodies against blood stage MSP-1(-43\%) and PE CSP (-28\%) antigens. However, the other nine PE antigens showed relative stability ( $-6 \%$ STD \pm 6$)$ as did the blood stage DBP antigen. This characteristic could be useful in defining serological markers of recent vs. historical exposure.

Even less well-studied than PE $P v$ antibodies are T cell responses to natural $P v$ infection. A few studies have assessed vaccine candidates eliciting $C D 8+$ and/or CD $4+T$ cells that correlate with protection from liver-stage infection in mice[32,33] and one clinical trial[34], but the mechanism that leads to T cell protection remains to be elucidated. Here, in a limited sample of volunteers, we observed a positive T cell response in $35 \%$ vs $9-35 \%$ of total individuals against blood stage antigen MSP1 and PE antigens, respectively. From patients with a positive $T$ cell response we observed that $40 \%$ showed reactivity for both blood stage and PE antigens which did not appear to correlate with IgG responses. While the role of T cell-mediated protection against rodent malaria liver stages is robust[35, 36], the absence of appropriate animal models for $P v$ and in particular hypnozoites leaves this possibility unconfirmed. However, recent evidence demonstrated that CD8 T cells can eliminate infected reticulocytes[37] opening the possibility of multi-stage CD8 T cell vaccines to prevent or limit $P v$ infection. Our limited data 
demonstrate that natural PE T cell immunity does exist and will need to be included in future studies investigating the relationship between $T$ cells and protection from infection at multiple stages.

Importantly, our A. nancymaae $P$. vivax model showed a predictable and consistent blood stage infection that can be immediately useful for testing new blood stage vaccine candidates or drugs against $P v$ blood stage infection. In addition to demonstrating an infection course similar in variation and magnitude observed in humans, the IgG antibody response also appeared to largely reflect our observations in humans. Specifically, a positive IgG response to one antigen was highly predictive of an IgG response to others. However, there were no animals which were negative for PE antigens as seen in our human samples perhaps due to more homogenous genetics or baseline states of our animals. Unlike humans, we saw no correlation between parasitemia and IgG response in our monkeys which could indicate a difference between humans and monkeys or simply reflect differences in sampling time points and the effect of previous exposure.

Our results in monkeys also demonstrate that blood stage infection on its own elicits antibodies that are reactive to PE antigens. Given the lack of evidence that these proteins are actually expressed at the blood stage, this cross-reactivity raises interesting considerations for the design of multi-stage $P$. vivax vaccines and interpretation of natural infection serology. In contrast to this cross-stage reactivity, monkeys infected with $P$. vivax or $P$. falciparum showed low cross-species reactivity against blood stage antigen MSP1 and no cross-reactivity against the PE antigen CSP. This also warrants further investigation as it could prove useful for serological surveys of malaria prevalence especially in co-endemic areas.

\section{Conclusion}

In conclusion, we present the most in-depth survey of PE immunity acquired during natural infection in the Peruvian Amazon. Our results demonstrate broad but variable reactivity across $P v P E$ antigens and between individuals. In addition, we find these results are largely replicated in a novel Aotus non-human primate $P V$ blood stage model which closely-reproduces human infection in terms of parasitology and IgG responses to blood stage and PE antigens. Together, these data clearly demonstrate a previously underappreciated prevalence of natural immunity to PE antigens which warrants specifically designed longitudinal cohorts where better correlations to transmission and protection from infection can be assessed. Such studies can add to a growing understanding of immunity to $P$. vivax infection in terms of both antigens and immune effector mechanisms with the goal of designing vaccines capable of preventing or reducing infection.

\section{Abbreviations}

Pv: Plasmodium vivax; PE: pre-erythrocytic; ELISA: enzyme-linked immunosorbent assay; $\boldsymbol{P f :} \boldsymbol{P}$ falciparum, MSP1: merozoite surface protein-1; DBP: Duffy Binding Protein; CSP: Circumsporozoite protein; WHO: World Health Organization; TRAP: thrombospondin-related adhesive protein; RH5: reticulocyte-binding protein Homolog 5; iRBCs: infected red blood cells; MQ: Mefloquine; CQ: Chloroquine; 
RAS: radiation attenuated sporozoites; GEST :egress and sporozoite traversal; ETRAMP: early transcribed membrane protein; AUC: Area Under the Curve; STD: standard deviation

\section{Declarations}

\section{Acknowledgments}

The authors are grateful to Dr. Danett Bishop, Dr. Christie Joya, Dr. Stephen Lizewski, and Dr. Hugo Valdivia for their support and critical review during the development of this work at NAMRU-6 and Dr. Simon Draper for his support for the use of monkey samples from RH5 studies (Jenner Institute, University of Oxford).

\section{Disclaimers}

"The views expressed in this article reflect the results of research conducted by the author and do not necessarily reflect the official policy or position of the Department of the Navy, Department of Defense, nor the United States Government"

\section{Authors' contributions}

GCB, JCA and BKW conceived, designed, and supervised the project studies. JVA, LLT, RP, AF and ML performed experiments of protein production and/or evaluation of immunogenicity by ELISA or ELISPOT. JVA, LLT and BKW performed the data and statistical analysis. JVA, LLT, LS and BKW wrote the first draft of the manuscript. JVA, LLT and BKW wrote the final version of the paper. All authors reviewed, edited and approved the manuscript.

\section{Funding}

This work was supported by advanced development funds LR233/Training grant NIH/FIC 2D43 TW007393 awarded to NAMRU-6 by the Fogarty International Center of the US National Institutes of Health and the Military Infectious Disease Research Program Grants \# F0398_14_LI, F0521_17 and F0578_19 awarded to Dr. G. Christian Baldeviano.

\section{Availability of data and materials}

All data generated or analyzed during this study are included in this published article.

\section{Ethics approval and consent to participate}

The human study protocols NMRCD.2007.0004, NMRCD.2010.0002 and NAMRU6.2012.0006 were approved by the U.S. Naval Medical Research Unit N ${ }^{\circ} 6$ Institutional Review Board in compliance with all applicable federal regulations governing the protection of human subjects 
The animal experiments reported herein were conducted in compliance with the Animal Welfare Act and in accordance with principles set forth in the "Guide for the Care and Use of Laboratory Animals," Institute of Laboratory Animals Resources, National Research Council, National Academy Press, 2011.

\section{Consent for publication}

Not applicable. No personal identifiable data was used in the study. All authors have provided consent to publish the findings in this study.

\section{Competing interests}

The authors declare that they have no competing interests.

\section{Copyright Statement}

"Several of the authors are members of federal/contracted employee of the United States government. This work was prepared as part of my official duties. Title 17 U.S.C. 105 provides that copyright protection under this title is not available for any work of the United States Government as part of that person's official duties"

\section{Author details}

${ }^{1}$ U.S. Naval Medical Research Unit No.6, Lima-Peru (NAMRU-6), ${ }^{2}$ Vysnova Partners Inc., ${ }^{3}$ NYC Department of Health and Mental Hygiene, ${ }^{4}$ U.S.Naval Medical Research Center (NMRC), ${ }^{5}$ Bluebird bio Inc.

\section{References}

1. WHO. World Malaria Report 2019. 2019.

2. Rahimi BA, Thakkinstian A, White NJ, Sirivichayakul C, Dondorp AM, Chokejindachai W. Severe vivax malaria: a systematic review and meta-analysis of clinical studies since 1900. Malar J. 2014;13:481.

3. Grillet ME, Hernandez-Villena JV, Llewellyn MS, Paniz-Mondolfi AE, Tami A, Vincenti-Gonzalez MF, Marquez M, Mogollon-Mendoza AC, Hernandez-Pereira CE, Plaza-Morr JD, et al: Venezuela's humanitarian crisis, resurgence of vector-borne diseases, and implications for spillover in the region. Lancet Infect Dis 2019.

4. White M, Amino R, Mueller I. Theoretical Implications of a Pre-Erythrocytic Plasmodium vivax Vaccine for Preventing Relapses. Trends Parasitol. 2017;33:260-3.

5. Tham WH, Beeson JG, Rayner JC. Plasmodium vivax vaccine research - we've only just begun. Int J Parasitol. 2017;47:111-8.

6. Regules JA, Cicatelli SB, Bennett JW, Paolino KM, Twomey PS, Moon JE, Kathcart AK, Hauns KD, Komisar JL, Qabar AN, et al. Fractional Third and Fourth Dose of RTS,S/AS01 Malaria Candidate Vaccine: A Phase 2a Controlled Human Malaria Parasite Infection and Immunogenicity Study. J Infect Dis. 2016;214:762-71. 
7. Olotu A, Fegan G, Wambua J, Nyangweso G, Leach A, Lievens M, Kaslow DC, Njuguna P, Marsh K, Bejon P. Seven-Year Efficacy of RTS,S/AS01 Malaria Vaccine among Young African Children. N Engl J Med. 2016;374:2519-29.

8. Sack B, Kappe SH, Sather DN. Towards functional antibody-based vaccines to prevent preerythrocytic malaria infection. Expert Rev Vaccines. 2017;16:403-14.

9. Douglas AD, Baldeviano GC, Jin J, Miura K, Diouf A, Zenonos ZA, Ventocilla JA, Silk SE, Marshall JM, Alanine DGW, et al: A defined mechanistic correlate of protection against Plasmodium falciparum malaria in non-human primates. Nat Commun 2019, 10:1953.

10. Douglas AD, Baldeviano GC, Lucas CM, Lugo-Roman LA, Crosnier C, Bartholdson SJ, Diouf A, Miura $\mathrm{K}$, Lambert LE, Ventocilla JA, et al. A PfRH5-based vaccine is efficacious against heterologous strain blood-stage Plasmodium falciparum infection in aotus monkeys. Cell Host Microbe. 2015;17:130-9.

11. Franca CT, He WQ, Gruszczyk J, Lim NT, Lin E, Kiniboro B, Siba PM, Tham WH, Mueller I. Plasmodium vivax Reticulocyte Binding Proteins Are Key Targets of Naturally Acquired Immunity in Young Papua New Guinean Children. PLoS Negl Trop Dis. 2016;10:e0005014.

12. King CL, Michon P, Shakri AR, Marcotty A, Stanisic D, Zimmerman PA, Cole-Tobian JL, Mueller I, Chitnis CE. Naturally acquired Duffy-binding protein-specific binding inhibitory antibodies confer protection from blood-stage Plasmodium vivax infection. Proc Natl Acad Sci U S A. 2008;105:83638.

13. Urusova D, Carias L, Huang Y, Nicolete VC, Popovici J, Roesch C, Salinas ND, Dechavanne S, Witkowski B, Ferreira MU, et al. Structural basis for neutralization of Plasmodium vivax by naturally acquired human antibodies that target DBP. Nat Microbiol. 2019;4:1486-96.

14. He WQ, Karl S, White MT, Nguitragool W, Monteiro W, Kuehn A, Gruszczyk J, Franca CT, Sattabongkot $\mathrm{J}$, Lacerda MVG, et al. Antibodies to Plasmodium vivax reticulocyte binding protein $2 \mathrm{~b}$ are associated with protection against P. vivax malaria in populations living in low malaria transmission regions of Brazil and Thailand. PLoS Negl Trop Dis. 2019;13:e0007596.

15. Longley RJ, White MT, Takashima E, Morita M, Kanoi BN, Li Wai Suen CSN, Betuela I, Kuehn A, Sripoorote P, Franca CT, et al. Naturally acquired antibody responses to more than 300 Plasmodium vivax proteins in three geographic regions. PLoS Negl Trop Dis. 2017;11:e0005888.

16. Franca CT, White MT, He WQ, Hostetler JB, Brewster J, Frato G, Malhotra I, Gruszczyk J, Huon C, Lin E, et al: Identification of highly-protective combinations of Plasmodium vivax recombinant proteins for vaccine development. Elife 2017, 6.

17. Molina DM, Finney OC, Arevalo-Herrera M, Herrera S, Felgner PL, Gardner MJ, Liang X, Wang R. Plasmodium vivax pre-erythrocytic-stage antigen discovery: exploiting naturally acquired humoral responses. Am J Trop Med Hyg. 2012;87:460-9.

18. Yadava A, Hall CE, Sullivan JS, Nace D, Williams T, Collins WE, Ockenhouse CF, Barnwell JW. Protective efficacy of a Plasmodium vivax circumsporozoite protein-based vaccine in Aotus nancymaae is associated with antibodies to the repeat region. PLoS Negl Trop Dis. 2014;8:e3268. 
19. Boonhok R, Rachaphaew N, Duangmanee A, Chobson P, Pattaradilokrat S, Utaisincharoen P, Sattabongkot J, Ponpuak M. LAP-like process as an immune mechanism downstream of IFNgamma in control of the human malaria Plasmodium vivax liver stage. Proc Natl Acad Sci U S A. 2016;113:E3519-28.

20. Carrasco-Escobar G, Gamboa D, Castro MC, Bangdiwala SI, Rodriguez H, Contreras-Mancilla J, Alava F, Speybroeck N, Lescano AG, Vinetz JM, et al. Micro-epidemiology and spatial heterogeneity of P. vivax parasitaemia in riverine communities of the Peruvian Amazon: A multilevel analysis. Sci Rep. 2017;7:8082.

21. Snounou G, Viriyakosol S, Zhu XP, Jarra W, Pinheiro L, do Rosario VE, Thaithong S, Brown KN. High sensitivity of detection of human malaria parasites by the use of nested polymerase chain reaction. Mol Biochem Parasitol. 1993;61:315-20.

22. Aguiar JC, Bolton J, Wanga J, Sacci JB, Iriko H, Mazeika JK, Han ET, Limbach K, Patterson NB, Sedegah M, et al. Discovery of Novel Plasmodium falciparum Pre-Erythrocytic Antigens for Vaccine Development. PLoS One. 2015;10:e0136109.

23. Epstein JE, Paolino KM, Richie TL, Sedegah M, Singer A, Ruben AJ, Chakravarty S, Stafford A, Ruck RC, Eappen AG, et al. Protection against Plasmodium falciparum malaria by PfSPZ Vaccine. JCI Insight. 2017;2:e89154.

24. Richie TL, Billingsley PF, Sim BK, James ER, Chakravarty S, Epstein JE, Lyke KE, Mordmuller B, Alonso P, Duffy PE, et al. Progress with Plasmodium falciparum sporozoite (PfSPZ)-based malaria vaccines. Vaccine. 2015;33:7452-61.

25. Limbach K, Aguiar J, Gowda K, Patterson N, Abot E, Sedegah M, Sacci J, Richie T. Identification of two new protective pre-erythrocytic malaria vaccine antigen candidates. Malar J. 2011;10:65.

26. Swearingen KE, Lindner SE, Flannery EL, Vaughan AM, Morrison RD, Patrapuvich R, Koepfli C, Muller I, Jex A, Moritz RL, et al. Proteogenomic analysis of the total and surface-exposed proteomes of Plasmodium vivax salivary gland sporozoites. PLoS Negl Trop Dis. 2017;11:e0005791.

27. Tsuboi T, Takeo S, Iriko H, Jin L, Tsuchimochi M, Matsuda S, Han ET, Otsuki H, Kaneko O, Sattabongkot $\mathrm{J}$, et al. Wheat germ cell-free system-based production of malaria proteins for discovery of novel vaccine candidates. Infect Immun. 2008;76:1702-8.

28. Longley RJ, Reyes-Sandoval A, Montoya-Diaz E, Dunachie S, Kumpitak C, Nguitragool W, Mueller I, Sattabongkot J. Acquisition and Longevity of Antibodies to Plasmodium vivax Preerythrocytic Antigens in Western Thailand. Clin Vaccine Immunol. 2016;23:117-24.

29. Matos ADS, Rodrigues-da-Silva RN, Soares IF, Baptista BO, de Souza RM, Bitencourt-Chaves L, Totino PRR, Sanchez-Arcila JC, Daniel-Ribeiro CT, Lopez-Camacho C, et al. Antibody Responses Against Plasmodium vivax TRAP Recombinant and Synthetic Antigens in Naturally Exposed Individuals From the Brazilian Amazon. Front Immunol. 2019;10:2230.

30. Keitany GJ, Kim KS, Krishnamurty AT, Hondowicz BD, Hahn WO, Dambrauskas N, Sather DN, Vaughan AM, Kappe SH, Pepper M. Blood Stage Malaria Disrupts Humoral Immunity to the Preerythrocytic Stage Circumsporozoite Protein. Cell Rep. 2016;17:3193-205. 
31. Kotliarov Y, Sparks R, Martins AJ, Mule MP, Lu Y, Goswami M, Kardava L, Banchereau R, Pascual V, Biancotto $A$, et al. Broad immune activation underlies shared set point signatures for vaccine responsiveness in healthy individuals and disease activity in patients with lupus. Nat Med. 2020;26:618-29.

32. Bauza K, Malinauskas T, Pfander C, Anar B, Jones EY, Billker O, Hill AV, Reyes-Sandoval A. Efficacy of a Plasmodium vivax malaria vaccine using ChAd63 and modified vaccinia Ankara expressing thrombospondin-related anonymous protein as assessed with transgenic Plasmodium berghei parasites. Infect Immun. 2014;82:1277-86.

33. Atcheson E, Bauza K, Salman AM, Alves E, Blight J, Viveros-Sandoval ME, Janse CJ, Khan SM, Hill AVS, Reyes-Sandoval A. Tailoring a Plasmodium vivax Vaccine To Enhance Efficacy through a Combination of a CSP Virus-Like Particle and TRAP Viral Vectors. Infect Immun 2018, 86.

34. Bennett JW, Yadava A, Tosh D, Sattabongkot J, Komisar J, Ware LA, McCarthy WF, Cowden JJ, Regules J, Spring MD, et al. Phase 1/2a Trial of Plasmodium vivax Malaria Vaccine Candidate VMP001/AS01B in Malaria-Naive Adults: Safety, Immunogenicity, and Efficacy. PLoS Negl Trop Dis. 2016;10:e0004423.

35. Schmidt NW, Butler NS, Badovinac VP, Harty JT. Extreme CD8 T cell requirements for anti-malarial liver-stage immunity following immunization with radiation attenuated sporozoites. PLoS Pathog. 2010;6:e1000998.

36. Parmar R, Patel H, Yadav N, Parikh R, Patel K, Mohankrishnan A, Bhurani V, Joshi U, Dalai SK. Infectious Sporozoites of Plasmodium berghei Effectively Activate Liver CD8alpha(+) Dendritic Cells. Front Immunol. 2018;9:192.

37. Junqueira C, Barbosa CRR, Costa PAC, Teixeira-Carvalho A, Castro G, Sen Santara S, Barbosa RP, Dotiwala F, Pereira DB, Antonelli LR, et al. Cytotoxic CD8(+) T cells recognize and kill Plasmodium vivax-infected reticulocytes. Nat Med. 2018;24:1330-6.

\section{Tables}

Table 1. Demographic information of $P$. vivax infected patients by site of sample collection. Differences of epidemiological variables between groups of sample collection were assessed using Mann-Whitney $U$ test and Chi-square test with significant values for $p<0.05$ and $p<0.001$. 


\begin{tabular}{lccc}
\hline Characteristics & $\begin{array}{c}\text { Hospital Apoyo } \\
\text { Iquitos (HAl) } \mathbf{n = 3 5}\end{array}$ & $\begin{array}{c}\text { Hospital regional } \\
\text { Loreto (HRL) } \mathbf{n = 4 1}\end{array}$ & p-value \\
\hline Age (Median, IQR) & 27 & 35 & 0.391 \\
Male sex (\%) & $(9-68)$ & $(8-88)$ & 0.309 \\
Asexual par/ul (Median, IQR) & 54 & 60 & 0.692 \\
Temperature C & & & \\
Weight Kg (Median, IQR) & 2,830 & 3,045 & 0.198 \\
Number of previous & $(36-14,130)$ & $(24-24,180)$ & \\
$\boldsymbol{P}$. vivax episodes (Median, IQR) & 37.8 & 37.0 & 0.037 \\
\hline
\end{tabular}

Table 2. Demographic information of $P$. vivax infected patients by low and high antibody responders. Epidemiological variables were compared between low and high responder group. Categorical variables were tested by Chi2 and numerical variables by Mann-Whitney $U$ test, significance was reported by $\mathrm{p}<$ 0.05 , and $p<0.001$.

Table 2. Demographic information of $P$. vivax infected patients by low and high antibody responders

\begin{tabular}{lccc}
\hline Characteristics & $\begin{array}{c}\text { low Ab responders } \\
\mathrm{n}=38\end{array}$ & $\begin{array}{c}\text { high Ab responders } \\
\mathrm{n}=38\end{array}$ & p-value \\
\hline Place-HRL (\%) & 44 & 56 & 0.250 \\
Place-HAI (\%) & 57 & 43 & 0.250 \\
Male sex (\%) & 49 & 51 & 0.817 \\
Age (Median, IQR) & 30 & 30 & 0.790 \\
& & & \\
Asexual par/ul (Median, IQR) & 4,663 & 1,873 & 0.008 \\
Temperature C ${ }^{\circ}$ (Median, IQR) & $(36-24,180)$ & $(24-12,572)$ & 0.468 \\
Weight Kg (Median, IQR) & 37.0 & 37.0 & \\
& $(36.0-41.5)$ & $(35.9-40.0)$ & 0.433 \\
Number of previous & 60.0 & 66.5 & \\
$\boldsymbol{P}$. vivax episodes (Median, IQR) & $(16-98)$ & $(29-90)$ & 0.172 \\
\hline
\end{tabular}

Table 3. Comparative analysis of antibody and T cell responders against blood stage and PE antigens. Prevalence of ELISA and ELISOPT was measured by IgG and Interferon gamma positivity in $P$. vivax infected individuals. 
Table 3. Paired ELISA and ELISPOT results of up to 7 subjects for each protein tested by both assays

\begin{tabular}{lcccccccc}
\hline Protein ID & PVX Code & $\begin{array}{c}\text { Parasite } \\
\text { stage }\end{array}$ & ELISA & ELISPOT + IgG+ / IFN+ & IgG+ / IFN- & IgG- / IFN+ & IgG- / IFN- \\
\hline MSP1 & PVX_099980 & Blood & $100 \%$ & $29 \%$ & $2 / 7$ & $5 / 7$ & $0 / 7$ & $0 / 7$ \\
\hline CSP & PVX_119355 & PE & $86 \%$ & $29 \%$ & $2 / 7$ & $4 / 7$ & $0 / 7$ & $1 / 7$ \\
CeITOS & PVX_123510 & PE & $57 \%$ & $29 \%$ & $2 / 7$ & $2 / 7$ & $0 / 7$ & $3 / 7$ \\
ETRAMP & PVX_121950 & PE & $50 \%$ & $33 \%$ & $1 / 6$ & $1 / 3$ & $1 / 6$ & $1 / 3$ \\
FALSTATIN & PVX_09903 & PE & $57 \%$ & $14 \%$ & $1 / 7$ & $3 / 7$ & $0 / 7$ & $3 / 7$ \\
Hypothetical & PVX_119755 & PE & $80 \%$ & $20 \%$ & $1 / 5$ & $3 / 5$ & $0 / 5$ & $1 / 5$ \\
HSP & PVX_089585 & PE & $100 \%$ & $0 \%$ & $0 / 1$ & $1 / 1$ & $0 / 1$ & $0 / 1$ \\
GEST & PVX_121950 & PE & $100 \%$ & $0 \%$ & $0 / 1$ & $1 / 1$ & $0 / 1$ & $0 / 1$ \\
\hline
\end{tabular}

Table 4. Parasitemia kinetics of $\boldsymbol{A}$. nancymaae monkeys infected with $P$. vivax Vietnam-IV strain.

Parasitemia was measured by microscopy and followed up from day 4 to 37 post infection with $P$. vivax Vietnam-IV. Monkeys were infected with dose of $2.5 \times 10^{6}$ par/animal (experiment 1) or $1.0 \times 10^{6}$ par/animal (experiment 2). Prepatency day (day to first parasitemia), day of maximum parasitemia and maximum parasitemia were determined by microscopy.

Table 4. Aotus nancymaae and $P$. vivax parasitemia information

\begin{tabular}{lcccc}
\hline Monkey ID & Experiment & $\begin{array}{c}\text { Prepatency } \\
\text { Day }\end{array}$ & $\begin{array}{c}\text { Day of maximun } \\
\text { parasitemia }\end{array}$ & $\begin{array}{c}\text { Maximun } \\
\text { parasitemia }\end{array}$ \\
\hline T3044 & 1 & 4 & 14 & 14600 \\
T3122 & 1 & 6 & 14 & 21600 \\
T3212 & 1 & 4 & 10 & 13000 \\
\hline T3242 & 2 & 5 & 11 & 2100 \\
T3251 & 2 & 7 & 11 & 29200 \\
T3215 & 2 & 7 & 15 & 13500 \\
T3237 & 2 & 7 & 13 & 7920 \\
T3244 & 2 & 5 & 15 & 3760 \\
T3308 & 2 & 5 & 17 & 194000 \\
\hline Exp 1 average & & $\mathbf{5}$ & $\mathbf{1 3}$ & $\mathbf{1 6 4 0 0}$ \\
STD & & 1 & 2 & 3735 \\
Exp 2 average & & $\mathbf{6}$ & $\mathbf{1 4}$ & $\mathbf{4 1 7 4 7}$ \\
STD & & 1 & 2 & 68672 \\
\hline
\end{tabular}

Table 5. Parasitemia kinetics of $A$. nancymaae monkeys infected with $P$. falciparum FVO strain. Parasitemia was measured by microscopy and followed up from day 3 to 30 post infection with $P$. falciparum FVO. Monkeys were infected with dose of $1.0 \times 10^{\wedge} 4$ par/animal for experiment 1 and 2 . Prepatency day, day of maximum parasitemia and maximum parasitemia were determined by microscopy results. 
Table 5. Aotus nancymaae and $\boldsymbol{P}$. falciparum parasitemia information

\begin{tabular}{lcccc}
\hline Monkey ID & Experiment & $\begin{array}{c}\text { Prepatency } \\
\text { Day }\end{array}$ & $\begin{array}{c}\text { Day of maximun } \\
\text { parasitemia }\end{array}$ & $\begin{array}{c}\text { Maximun } \\
\text { parasitemia }\end{array}$ \\
\hline T2736 & 1 & 7 & 12 & 365444 \\
T2758 & 1 & 5 & 12 & 382500 \\
T2815 & 1 & 7 & 12 & 321879 \\
T2825 & 1 & 7 & 14 & 231250 \\
T2844 & 1 & 7 & 10 & 214700 \\
T2875 & 1 & 5 & 12 & 530462 \\
\hline T3409 & 2 & 5 & 10 & 223600 \\
T3427 & 2 & 7 & 11 & 283050 \\
T3473 & 2 & 6 & 11 & 769500 \\
T3496 & 2 & 6 & 11 & 385400 \\
\hline Exp 1 average & & 6 & 12 & 341039 \\
STD & & 1 & 1 & 105339 \\
Exp 2 average & & $\mathbf{6}$ & $\mathbf{1 1}$ & $\mathbf{4 1 5 3 8 8}$ \\
STD & & 1 & 1 & 212480 \\
\hline
\end{tabular}

\section{Supplementary}

Figure $\mathrm{S} 1$ is not available with this version

Figures 


\section{Antigen Figure}

\section{- Pv(-) \\ $\square \operatorname{Pv}(+)$ \\ - (+) cutoff}

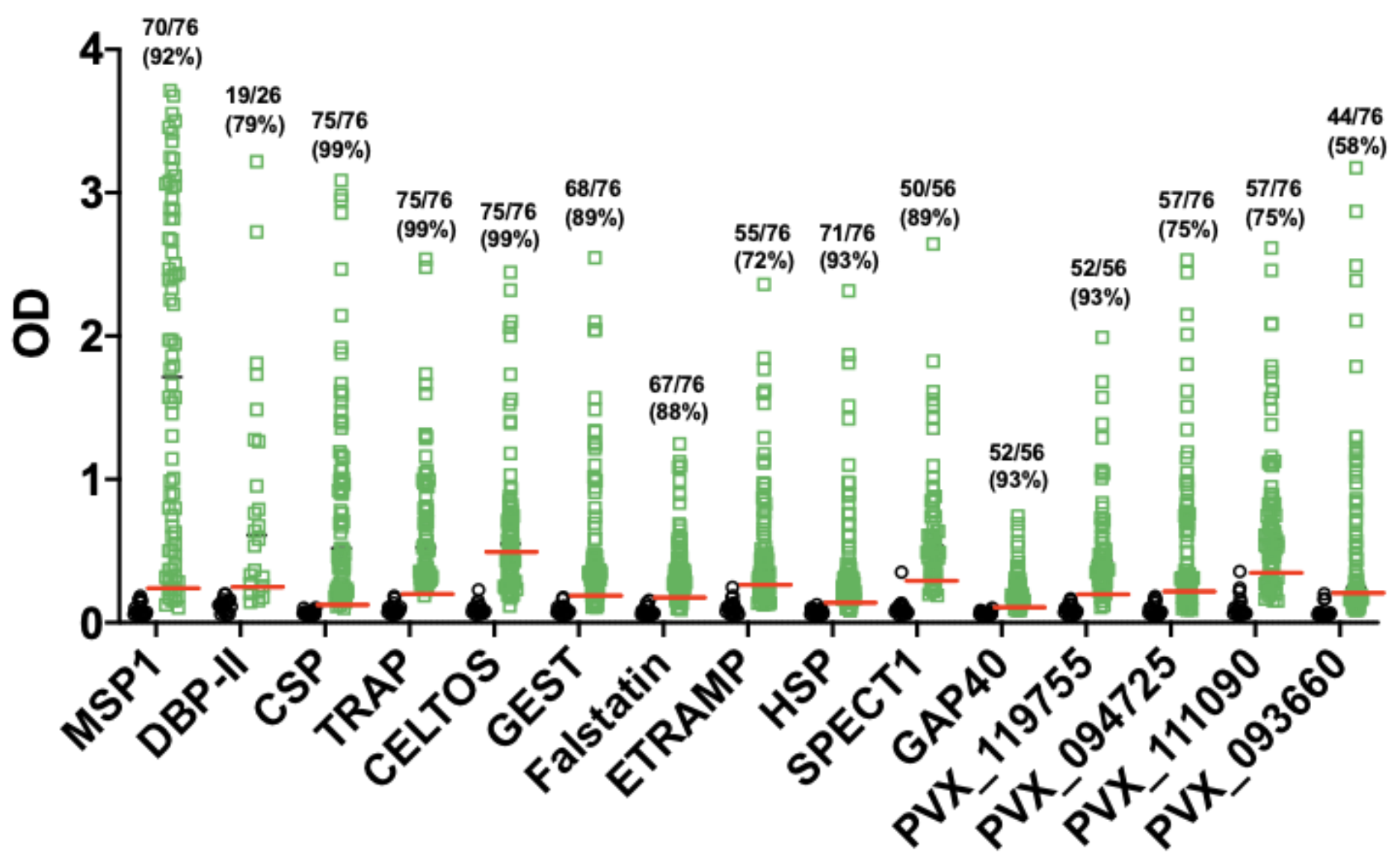

Antigen

Figure 1

Prevalence against $P$. vivax PE antigens in P. vivax infected Peruvian population. Plasma samples from healthy controls (black circles) and P. vivax patients (green squares) were tested against 15 P. vivax antigens to determine seroprevalence by ELISA. Data shows individual values of IgG antibodies against each antigen measured by OD values. OD positivity cut-off (red line) value was defined as the average of low endemic control samples plus three standard deviations per each antigen. 


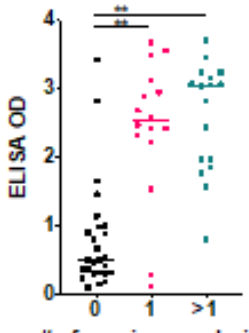

\# of previous malaria episodes

GE ST

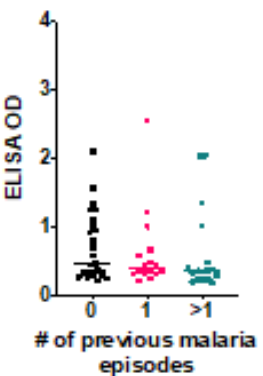

GAP40

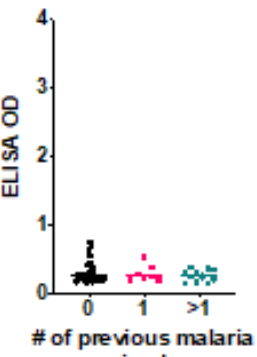

episodes
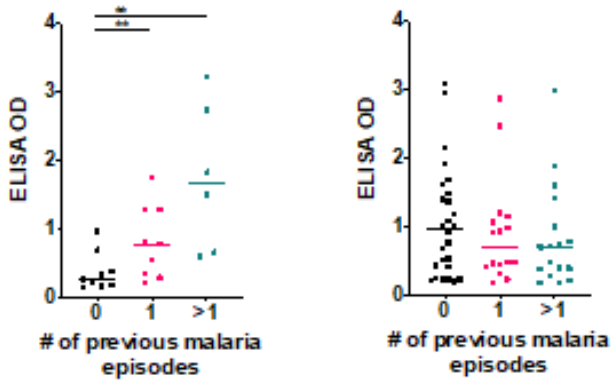

episodes

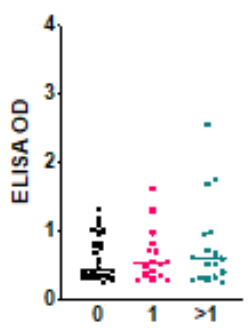

\# of previous malaria episodes

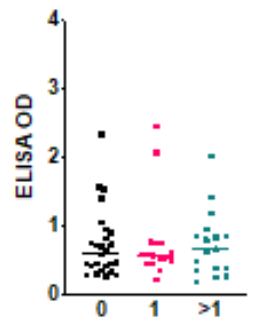

\# of previous malaria episodes
F alstatin

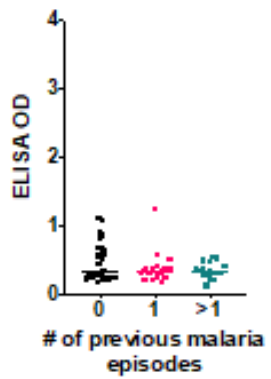

PVX_119755

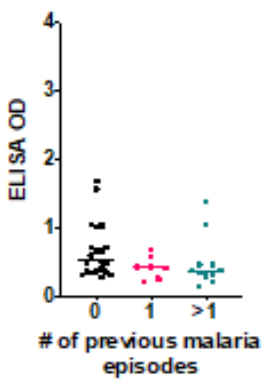

ETRAMP

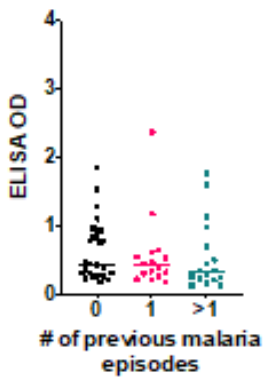

PVX_094725

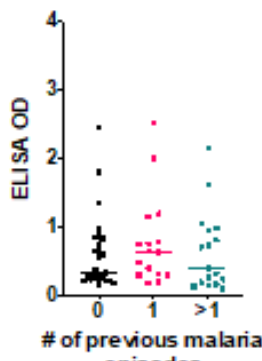
episodes
H SP

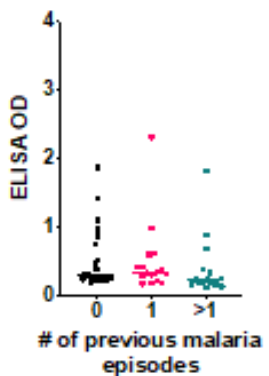

episodes
SPECT1

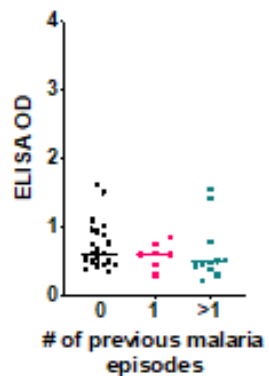

\section{Figure 2}

Boosting effect of blood and pre-erythrocytic P. vivax antigens in P. vivax infected Peruvian population. Plasma samples from zero previous $(n=9-26)$, one previous $(n=7-16)$ and two or more previous $(n=6-17) P$. vivax episodes were used to determine boosting effect against each P. vivax antigen. Dot plots represent P. vivax episode groups with OD values of IgG antibodies against each antigen. Differences between 
groups per each antigen were assessed using the Kruskal-Wallis test with Mann-Whitney U post-test. * $p<$ 0.05 , and $* \star p<0.001$.

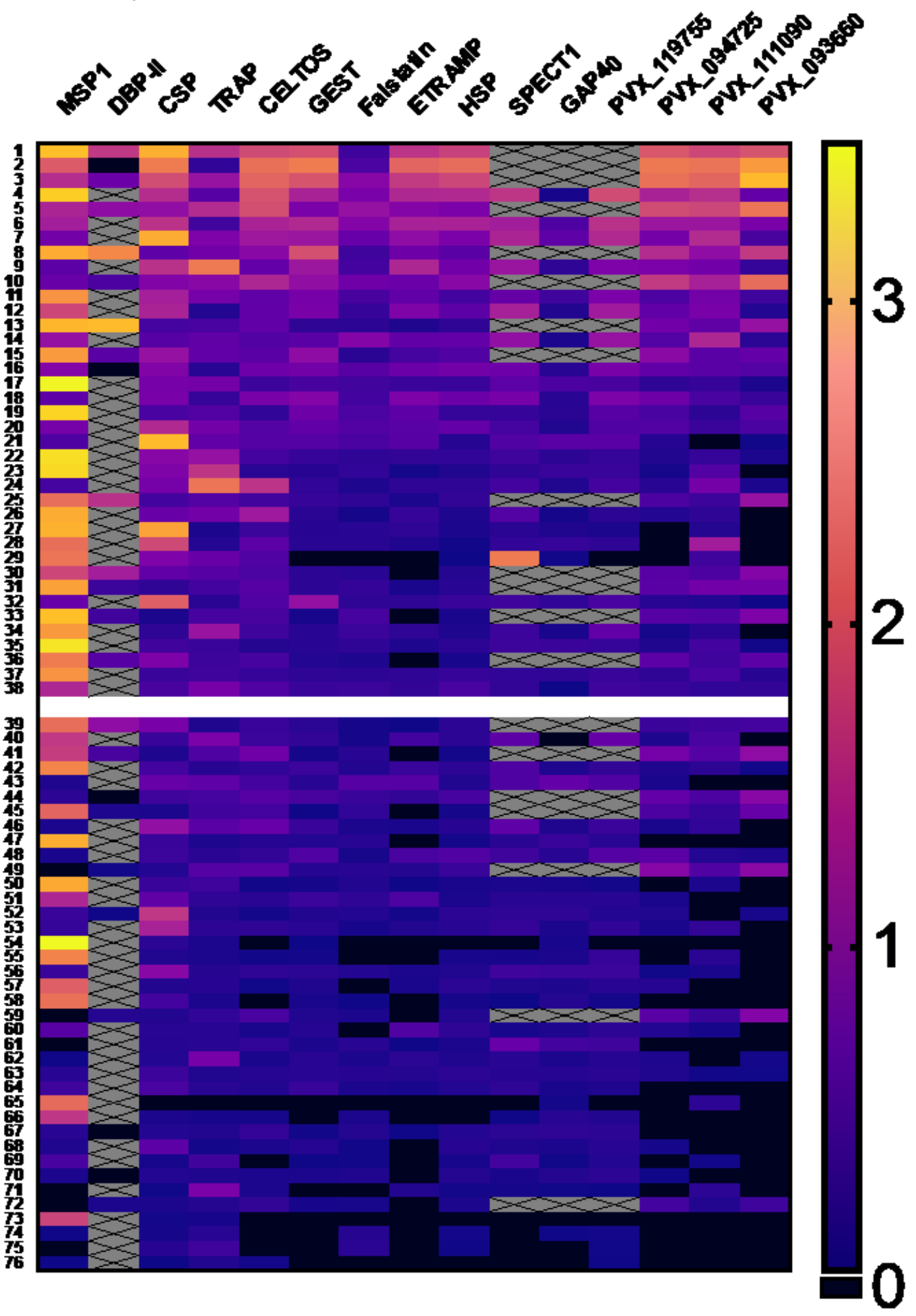

Figure 3

Reactivity of pre-erythrocytic P. vivax antigens in P. vivax infected Peruvian population. Individual reactivity against each $P$. vivax antigen is shown by OD. Negative reactivity is shown as 0 (dark blue) and positive reactivity with OD values between 0.2-3.5 (blue to yellow). Volunteers are arranged in order of 
descending total antibody response across all antigens with the gap showing the division between "high responders" and "low responders". Boxes with " $X$ " indicate samples not measured due to either limited sample availability or inability to finish analysis due to the COVID-19 pandemic.
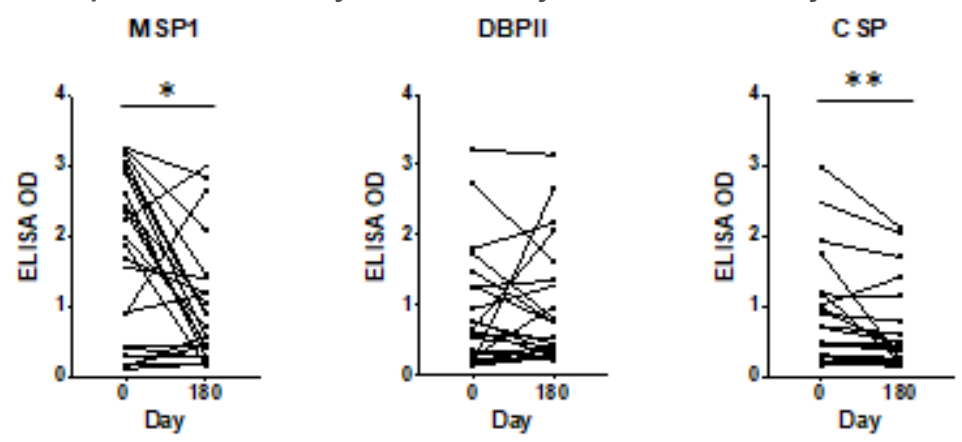

ETRAMP
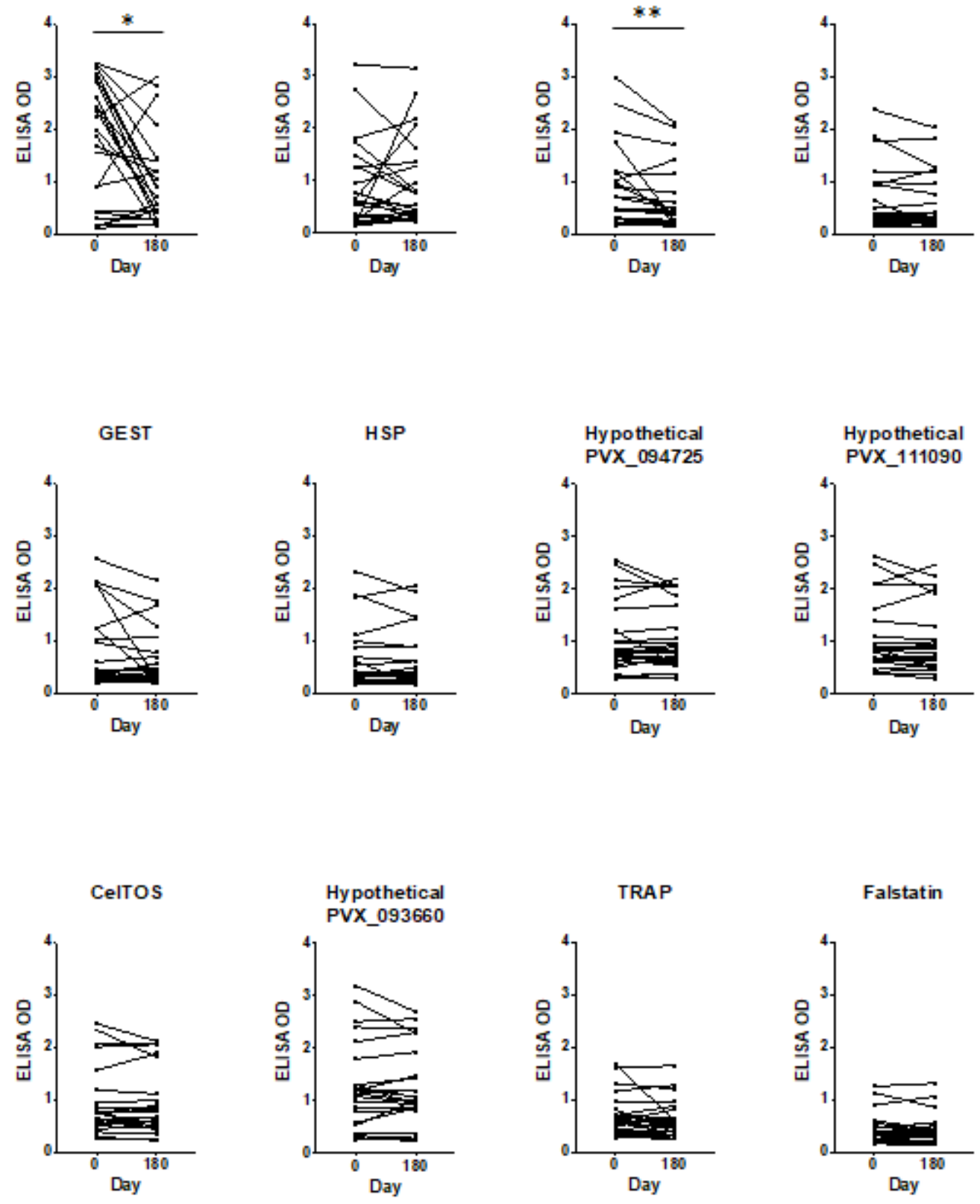


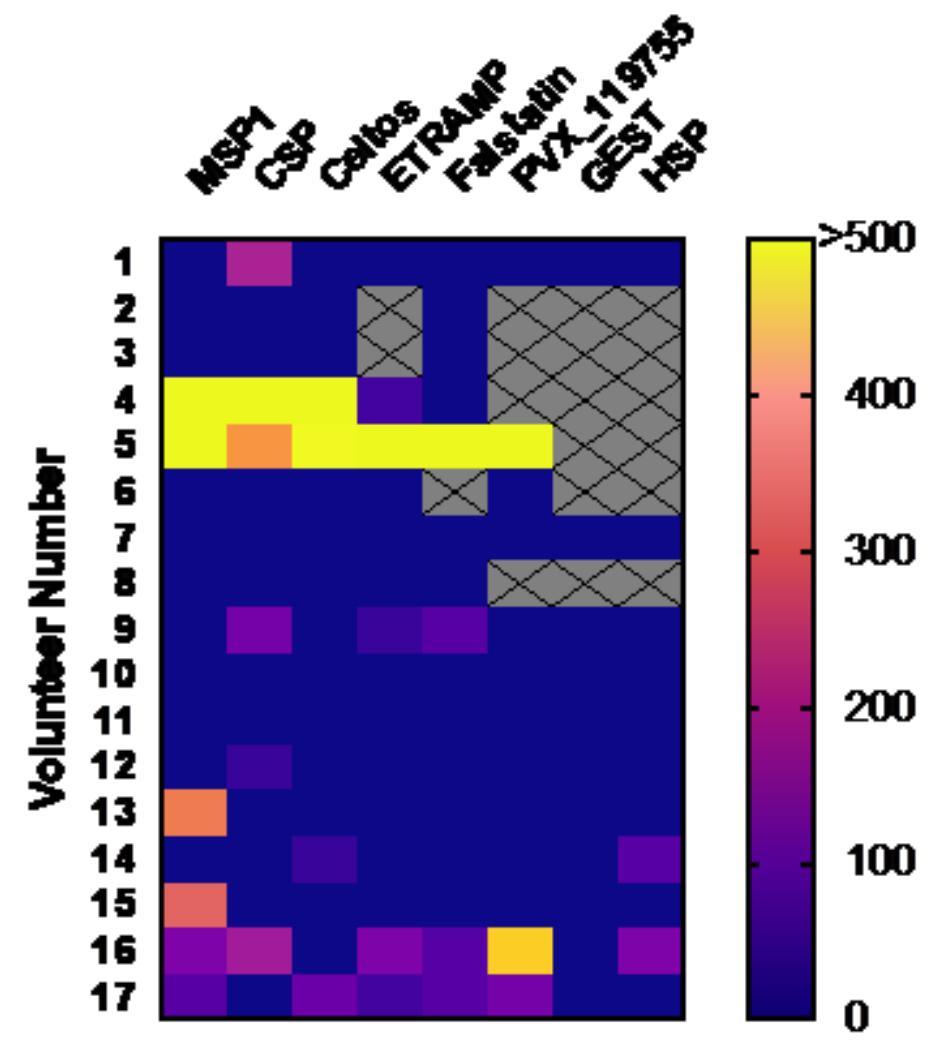

Figure 5

IFN- $\gamma$ response to seven PE P. vivax antigens evaluated in 17 malaria patients from lquitos. Shown as SFU/106 PBMCs for samples which were $>20 \%$ above negative controls after subtracting background. Boxes with " $X$ " indicate samples not measured due limited sample availability. 
A

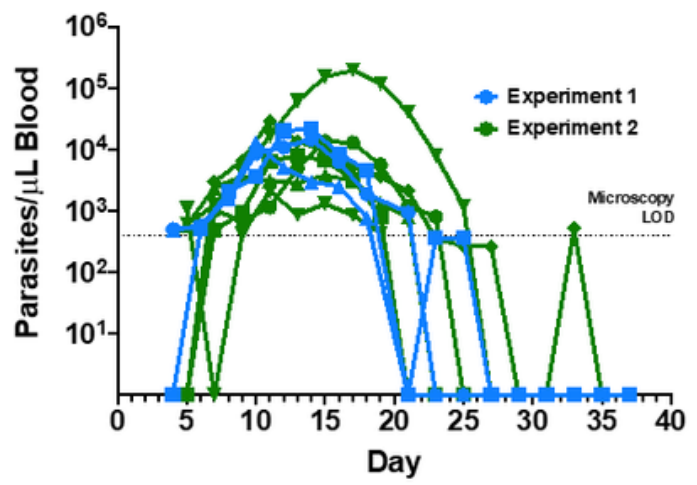

C Par AUC vs. AUC OD

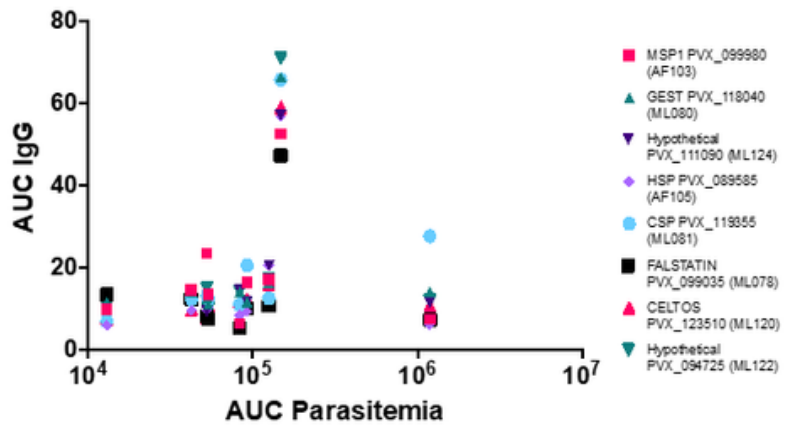

B

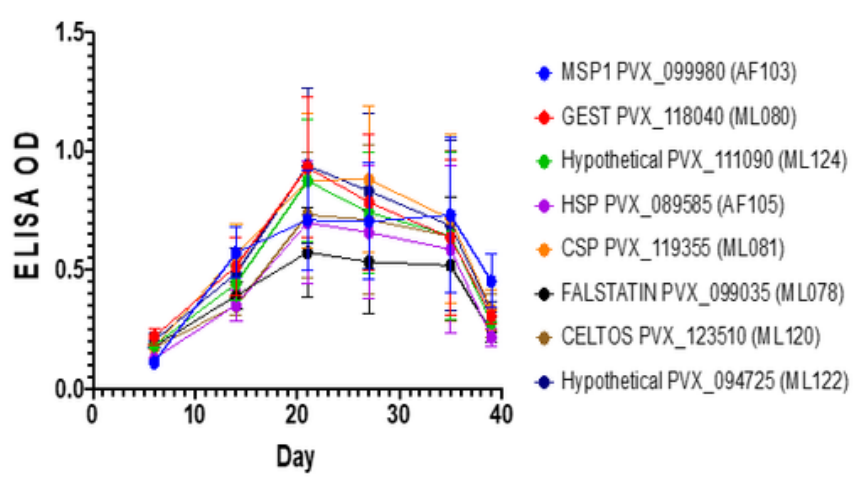

D Par Peak vs. AUC OD

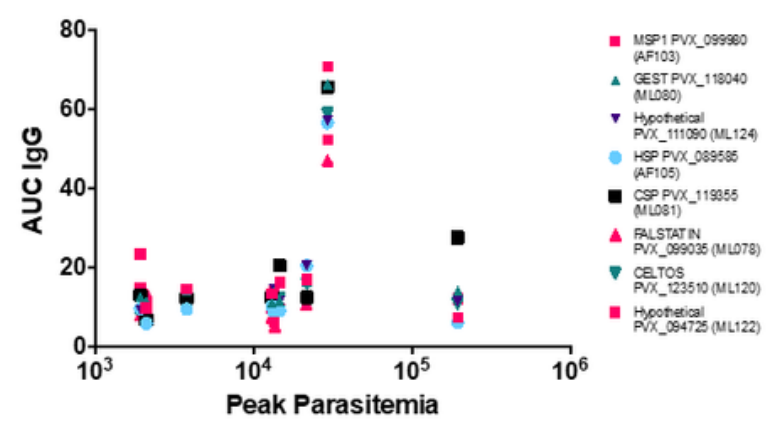

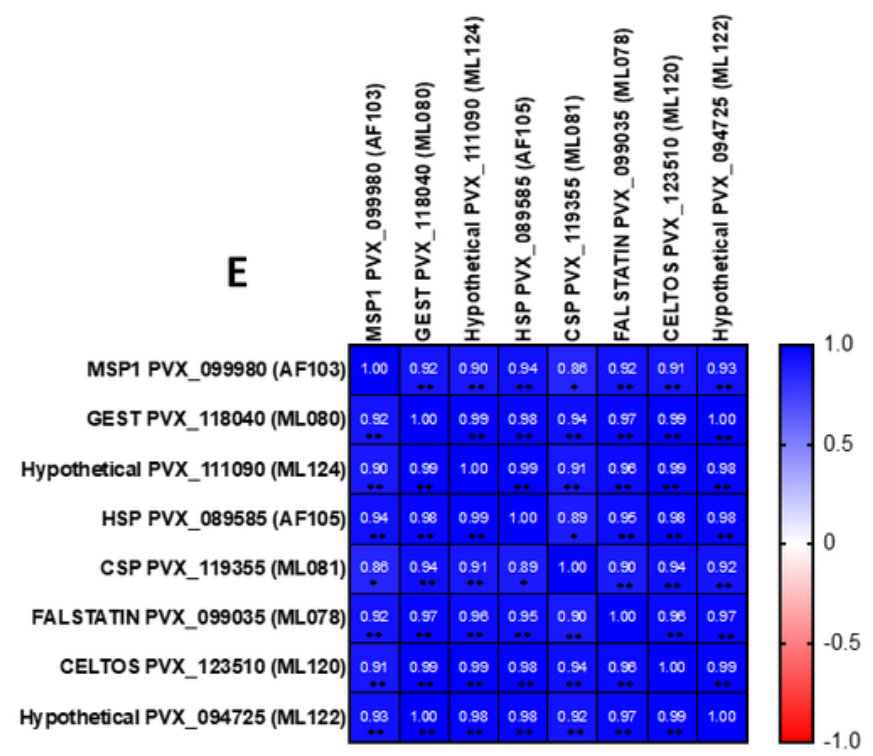

Figure 6

Parasitemia kinetics and humoral immune response against $P$. vivax Blood stage and PE antigens of $A$. nancymaae monkeys infected with P. vivax Vietnam-IV strain. (A) Parasitemia kinetics of monkeys infected with $2.5 \times 106 \mathrm{par} /$ animal (experiment 1, blue lines) or $1.0 \times 10^{\wedge} 6 \mathrm{par} /$ animal (experiment 2, green lines). (B) Mean IgG antibodies for each antigen over the course of infection as measured by ELISA. Parasitemia levels vs. IgG antibodies were compared using the area under the curve during the entire 
infection period in (C) or the day of peak parasitemia in (D) for each antigen. (E) Correlation of IgG antibody response between proteins were measured by Pearson's correlation. Coefficients were represented with values of -1.0 to 1.0 , with colors red for negative correlation and blue for positive correlation. Significant correlations were represented by ${ }^{*} \mathrm{p}<0.05$ and ${ }^{*} \mathrm{p}<0.001$.
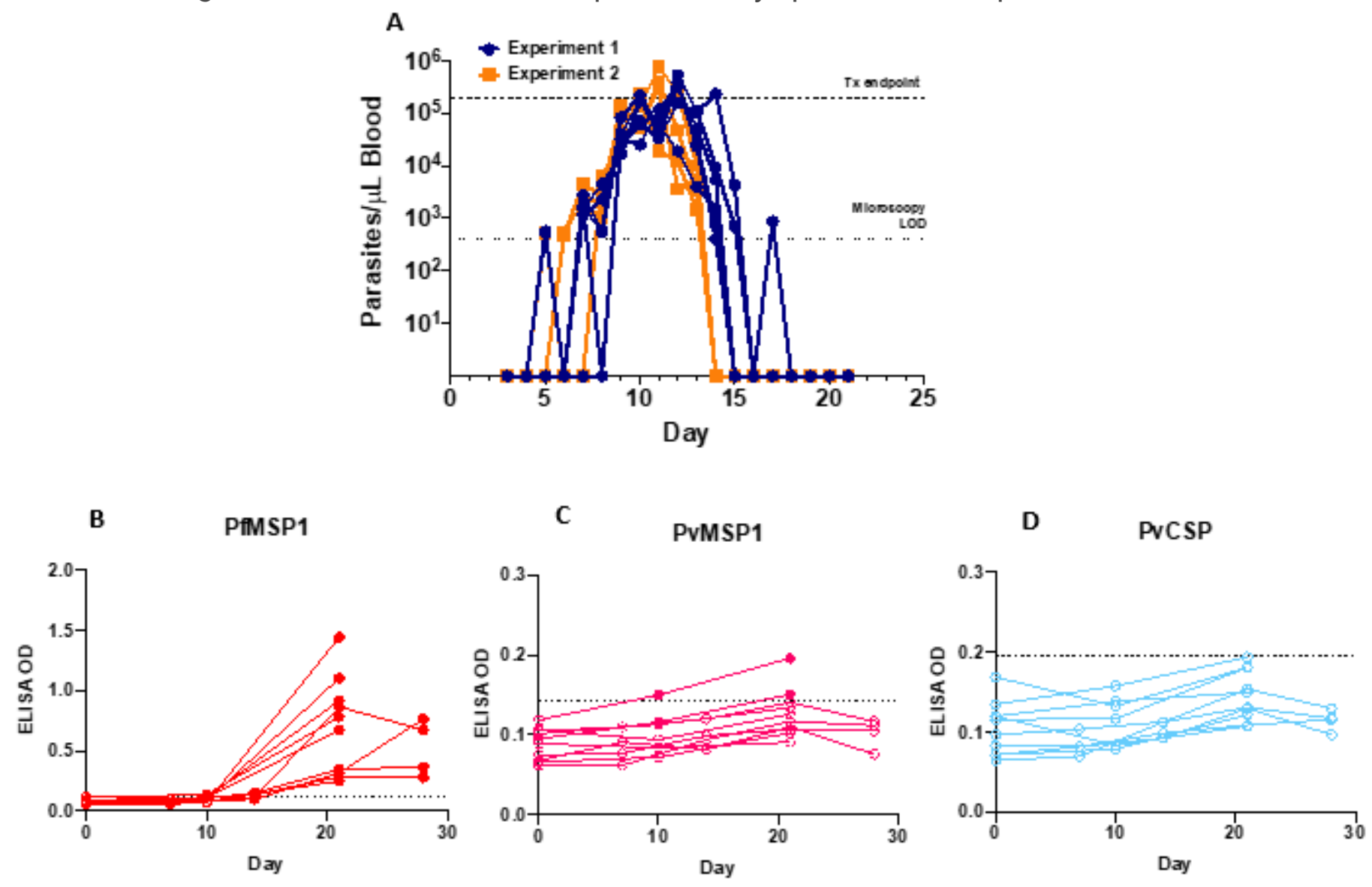

Figure 7

Parasitemia kinetics and humoral immune response against $P$. vivax and $P$. falciparum antigens of $A$. nancymaae monkeys infected with P. falciparum FVO strain. (A) Monkeys were infected with $1.0 \times 104$ iRBC/animal for experiment 1 (Blue lines) and experiment 2 (Orange lines). Parasitemia was measured by microscopy and followed up from day 3 to 30 post infection. IgG antibody responses against $P$. falciparum MSP1 antigen (B), P. vivax MSP1 antigen (C) and P. vivax CSP antigen (D) were measured by ELISA with individual animals shown with connecting lines. Dashed lines represent cutoff values for each antigen calculated by the average of OD values of uninfected animal plasma plus 3 standard deviations. Solid data points represent any values above this cutoff.

\section{Supplementary Files}

This is a list of supplementary files associated with this preprint. Click to download.

- TableS1.docx 
- S2.png

- S3.png

- S4.png

Page 28/28 